\title{
BOREL ON DIVERGENT SERIES
}

Leçons sur les Séries Divergentes. Par Émile Borel. Deuxième édition, revue et entièrement remaniée avec le concours de Georges Bouligand. Paris, Gauthier-Villars, 1928. vi +260 pp.

Those who have followed the progress made in the theory of divergent series during the past quarter of a century are well aware of the important rôle which the first edition of Professor Borel's book has played in stimulating the development of this theory. This was due, not only to the importance of the results contained in it, but also to the elegance of the exposition and the many suggestive lines of thought that were indicated.

Throughout the period referred to Professor Borel's book has remained a classic in its field, but in view of the rapid growth of the theory it was inevitable that certain portions of the discussion should seem somewhat lacking in completeness after a lapse of twenty-five years. It was therefore quite appropriate that a second edition should be issued, bringing up to date the sections where later research had thrown fuller light on the questions involved, and giving a succinct resumé of the many important developments in various branches of the theory that have been made since the date of the first edition.

The account of recent work is for the most part contained in an additional chapter of some thirty-five pages at the close of the volume. Considering the extent of the literature involved, ${ }^{*}$ it was obviously somewhat of a task to give a coherent account of it in such brief space. The difficulties of the undertaking have been overcome in admirable fashion, and while necessarily many important studies had to be dismissed with merely a reference, the chapter on the whole gives an excellent bird's-eye view of the research material with which it deals. By selection of certain central lines of thought that have characterized the research in question, it has been possible to unify many separate studies and thus make a considerable gain in the brevity and directness of their treatment.

Theoretical discussions of divergent series can be broadly classified in groups in accordance with their relationship to two fundamental methods of procedure. The methods in question may be designated briefly as follows: (1) summation by means; (2) summation by convergence factors. In many studies one or the other of these methods is used exclusively; others involve both of them, and some deal explicitly with various relationships between the two methods. In the first edition of the Leçons summation by means played the dominating rôle, and naturally the same thing is

* In Smail's History and Synopsis of the Theory of Summable Infinite Processes, which deals with work through the year 1922, there are listed more than 250 publications since the appearance of the first edition of Borel's book. The synoptic account of these books and memoirs, in most cases quite brief, occupies more than 150 pages. 
true of that part of the present edition which constitutes a revision of the first edition. However, in Chapter III, which deals with the general theory of summable series, attention is called at the beginning to these two methods. This is done in connection with an illustrative discussion of the application of the methods of divergent series to the study of Fourier's series. No better introduction to the two points of view could have been selected, since the application to Fourier's series furnishes a simple and illuminating example of the efficiency of the two methods and the interplay between them.

In the added chapter of the present work, Chapter VI, a condensed account is given of a variety of studies which center about the method of convergence factors. As some of the most fundamental work of recent years based on the method of means has already been treated in Chapter III, the latter method is more briefly dealt with here. In the field of applications of divergent series, the account is limited in the main to work in connection with Dirichlet's series, factorial series, and quasi-analytic functions.

Of the chapters that occur in the first edition, Chapter V, dealing with developments in series of polynomials, is carried over unchanged. The reason for this is well stated in a footnote by Professor Bouligand, who remarks that the chapter was not merely of a didactic character, but exhibited the contact of ideas of two great mathematicians.* Of the remaining chapters, those dealing with asymptotic series, continued fractions and the theory of Stieltjes, and analytic extension by the method of Borel summability cover practically the same ground as before. Many sections have been revised and certain amplifications and modifications suggested by later researches have been made, but the subject matter is substantially the same.

The chapter on the theory of summable series contains much new material, being about twice the size of the corresponding chapter in the first edition. Among the new topics introduced, in addition to the discussion of Fourier's series referred to above, may be mentioned a more complete discussion of the means of Cesàro and $\mathrm{H} i$ lder, including a proof of their equivalence, and some work of Sannia and Le Roy connected with Borel summability.

The final chapter of the book is followed by an interesting note by Professor Bouligand, demonstrating the impossibility of securing analytic extension by the use of means of certain general types. This note is followed by a list of results culled from various memoirs on divergent series and integrals, stated in the form of exercises.

C. N. MOORE

* Mittag-Leffler and Borel. 